\title{
Economic Analysis of HCV Different Screening Algorithms in Egypt
}

Amal S. Sedrak ${ }^{1}$, Amany A. Salem ${ }^{1}$, Mohamed Hassany ${ }^{2}$, Eman H. Elsebaie ${ }^{1}$

1. Public Health \&Community Medicine Department, Faculty of Medicine, Cairo University,Egypt

2.National Hepatology \& Tropical Medicine Research Institute,Egypt

Corresponding author: Eman Hany Ahmed Elsebaie, Email: eman.elsebaei@kasralainy.edu.eg Tel: 01005645129

\begin{abstract}
Background: A top ranked public health problems especially in developing countries is hepatitis $\mathrm{C}$ virus infection. Being asymptomatic infection, screening has been proposed as a credible public health strategy. Egyptian national guidelines were emphasizing on adopting screening programs nationwide. No economic evaluation studies tackling HCV screening based on risk exposure was conducted in Egypt so far.
\end{abstract}

Aim: To evaluate the cost effectiveness of screening algorithms among two different population subgroups.

Methods: A Cost-Utility analysis was conducted using a validated decision tree model linked to a Markov Model, to compare the Incremental Cost-Effectiveness Ratio (ICER) of two policy scenarios: No screening versus Screen-and-treat with direct-acting antiviral agents (DAA) ON two Egyptian population subgroups (high risk populations of acquiring HCV infection versus the overall population).

Results: The ICER for implementing screening on high-risk populations was 3895.31 EGY/ QALY, considered costeffective (below the Egyptian Threshold 46000 EGY/ QALY). Conclusion: The ICER for implementing screening on highrisk populations was cost-effective. Whereas concerning the general population, it is considered cost-saving strategy.

Keywords: Cost-effectiveness, economic evaluation, Hepatitis C, screening.

\section{INTRODUCTION}

Globally, Viral hepatitis is considered a serious public health problem, ranking the 7 th chief cause of death ${ }^{(1)}$. Hepatitis $\mathrm{C}$ virus (HCV) infection contributes to almost half of this mortality ${ }^{(2,3)}$. Worldwide, it is estimated that 170 million individuals and approximately 350 thousands patients, die annually from HCV-related diseases ${ }^{(4)}$. The progressive nature of HCV infection, is emphasized with the high global prevalence of cirrhosis and hepatocellular carcinoma (HCC), 27\% and 25\% respectively ${ }^{(5)}$. Despite the global prevalence of $\mathrm{HCV}$ worldwidem, countries as Egypt, Cameroon and Mongolia, report the uppermost HCV prevalence more than $10 \%{ }^{(6)}$. Egypt Demographic and Health Surveys (EDHS), conveyed a reducing in anti-HCV antibodies prevalence among 15-59 years aged population, in 2009 compared to 2015 , estimated to be $14.7 \%$ versus $10 \%$ respectively ${ }^{(7)}$, that is substantially higher than global levels ${ }^{(2,3)}$. Given the asymptomatic nature of most chronic HCV infections, early detection of cases ( during the long pre-symptomatic period) will allow patients to receive treatment before developing chronic complications ${ }^{(8)}$. In the same context, the existence of oral direct-acting antivirals (DAAs), with considerable efficacy, affords a priceless oppurtunity for dropping the epidemiological burden of $\mathrm{HCV}$ disease as well as its transmission ${ }^{(4,9)}$. Thus, complete cure of Hepatitis C could be achieved within 3 months using direct-acting antivirals (DAAs) ${ }^{(10)}$. World Health Organization (WHO) has recently published the 'Global Health Sector Strategy on Viral Hepatitis 2016-2021 (11), which accentuated, achieving service coverage targets to eliminate $\mathrm{HCV}$, a prominent public health threat by $2030^{(11,12)}$. The 2020 targets have been set to increase the number of diagnosed tests performed for hepatitis by $100 \%$ and to test $80 \%$ of all health care workers for $\mathrm{HBV}$ and $\mathrm{HCV}{ }^{(12)}$. WHO is determined to ensure the affordability and accessibility of DAAs particural for those in need. Dramatic dropping of prices was facilitated by introducing generic versions of these medicines (specially in a group of high-burden, low- middle-income countries) ${ }^{(10)}$. In this context, Egypt has initatied a national strategy for HCV control and implemented HCV prevention and treatment programs (13). Egypt ambitious national HCV treatment program, was launched following successful negotiations for $99 \%$ discounted DAAs prices ${ }^{(14) .}$ The treatment program is planning to treat over 250,000 chronic $\mathrm{HCV}$ infected patients per year, targeting to achieve a prevalence (chronic infection) of less than $2 \%$ by $2025^{(15)}$. On contrary, existing evidence suggests persistence of higher incidence levels in Egypt, than other countries, due to continuing $\mathrm{HCV}$ transmission ${ }^{(9)}$. There is scarcity of studies estimating the economic impact of HCV screening followed by treatment particurlarly in countries with high prevalence. Earlier economic analyses have mostly focused on the U.S. population recommended targeting high-risk population HCV screening, rather than, general population screening. This could be reasonable with low prevalence of $\mathrm{HCV}$ infections in United States $(16,17)$. Thus, anticipating the need for supporting evidence for policymakers on the health and economic consequences of hepatitis $\mathrm{C}$ screening, this research was aimed at evaluating screening algorithms among different population groups as proposed by Egypt national plan, in order to integrate the highest cost-effective HCV screening strategy in the Egyptian National plan of HCV 
control. Moreover, this should support selected researchers' capacity building on preparing a policy Brief highlighting the adopting of the highest cost-effective HCV screening program.

\section{AIM OF THE STUDY}

The study aimed to evaluate the cost-effectiveness of adopting a single screening program for hepatitis $\mathrm{C}$ among different population strata (based on their risk probability of acquiring $\mathrm{HCV}$ infection).

\section{Specific Objectives:}

1. Construct a Markov model to follow-up the two cohorts of apparently healthy individuals (with different HCV risk probabilities) through transitional health states till death (from liver-related causes) after diagnosis by the screening program.

2. Calculate Incremental Cost-Effectiveness Ratio (ICER) of adopting the screening program on the two cohorts of the population.

\section{SUBJECTS AND METHODS}

Study Design: A Full economic evaluation cost-utility analysis study was conducted using a tailored, validated decision tree model connected to Markov Model to compare the incremental Cost-Effectiveness Ratio (ICER) of two policy scenarios (examining 2 screening regimens):

1-No screening, 2- Screening then treatment with directacting antiviral agents (DAA) ON two Egyptian population subgroups (categorized according to their risk of acquiring $\mathrm{HCV}$ infection):

Group I: High risk populations: include populations with recurrent medical injections, blood transfusions e.g. hemophilia, hemodialysis, thalassemia, and people who inject drugs (PWID), among others ${ }^{(18)}$. Group II: General population: pretty low risk populations of exposure to HCV e.g. pregnants, blood donors, healthy children, outpatient clinic attendees.

Sampling and study population: Two hypothetical cohorts of 1000 individuals, each, presumed to be unconscious of their HCV infection state. Cost-utility of Screening versus No screening among the 2 cohorts was evaluated using a decision tree model.

A. Screening then treating with DAA: Screening is performed by a blood test for HCV antibody. An HCV RNA test will follow all positive antibody tests to confirm infection. Our analysis proposed that all tested positive individuals for both tests will be referred to a hepatologist to be offered treatment according to the Egyptian guidelines ${ }^{(19)}$. Treatment and monitoring for CHC: According to the Egyptian guidelines, diagnosed patients were categorized as either eligible to treatment or ineligible to treatment, and in turn, eligible patients were further classified into easy to treat group or difficult to treat group as illustrated in figure (2) ${ }^{(19)}$. This classification significantly varies in the treatment prognosis. After treatment, patients who achieve No SVR
(Sustained Virological Response) were 0.035 among difficult to treat group, versus 0.073 among easy to treat group ${ }^{(20)}$ with more probability of progressing to cirrhotic decompensated liver disease and HCC. Monitoring of $\mathrm{HCV}$ : viral load estimation is required at any time-point between 12 and 24 weeks' post-treatment to confirm the virus's successful eradication.

B. NON-screening, where an accidental diagnosis: is assumed to happen in merely $5 \%$ of the population ${ }^{(19)}$ while the rest seeks medical advice when complications appear (Cirrhosis, decompensated liver cirrhosis or HCC). We assumed that $100 \%$ of undiagnosed chronic infected patients were unaware of their situation.

The decision tree (for each policy scenario) is connected to a Markov Process model to forcast patients' outcomes (Figure 1). The model structure followed the natural history of the disease. The model cycle length was 1 year to allow for an accurate estimation of the different health states' timing and related costs ${ }^{(21)}$.

Study setting: The National Liver Institute was the site of data collection of model inputs in addition to holding the capacity building workshop for preparing a policy brief . Data collection methods: The study was conducted in 4 Phases:

Phase I: Extensive literature searches together with critical appraisal of relevant studies (principally published systematic reviews or meta-analyses of randomized controlled trials, cohort studies) to construct the decision Tree and the related Markov model, and perform extraction of model parameters. The extensive literature search was conducted on Medline and Google scholar databases for English articles published in the last 5 years to recover the available published data regarding the different health states' probabilities, SVR rates of therapy combinations, and the quality of life of the different health states ${ }^{(22,23)}$. Published Egyptian guidelines were used for different treatment scenarios ${ }^{(19)}$.

Phase II: Determination of primary outcomes, Total (QALYs) accrued per patient

\section{- Health Outcomes}

The health outcomes of each intervention were assessed in terms of quality-adjusted life-years (QALYs). This generic measurement assesses the length the patient lives by the quality of life while in a specific health state. Hence, QALYs combine both morbidity and mortality into a single parameter. The utility value of F3, F4, DCC, HCC, and post-LT health states besides the disutility value of the SOF $\mathrm{p}$ LDV regimen that were considered in the model that was taken from a decision-tree model that calculated the cost-utility of boceprevir $\mathrm{b}$ RBV $\mathrm{p}$ peg IFN compared to that of SOF $\mathrm{p}$ pegIFN $\mathrm{p}$ RBV and three peg IFN-free regimens (SOF $\mathrm{p}$ simeprevir, SOF $\mathrm{p} \mathrm{DCV}$, and SOF $\mathrm{p} \mathrm{LDV}$ ) in treatment-naive patients with chronic HCVgenotype ${ }^{(22)}$. 
Utility score between 0 (death) and 1 (perfect health) was assigned to every health state in the model, thus we can calculate patient utilities for the cohort present in that state. The utility measure of the L.T. health state, incorporated into the model, was derived from an observational, cross-sectional cohort study of 751 patients with HCV from several tertiary care settings in Canada to evaluate health-related quality of life across the HCV disease spectrum. This process was implemented using utility and psychometric methods, adjusted for sociodemographic aspects and comorbidities ${ }^{(24)}$.

Utility decrements that have been specific to each treatment regimen were allocated during treatment accounting for the diminshed health-related quality of life associated with treatment-related adverse events. Utility decrements during the treatment course were derived from a study that estimated the utility scores associated with treatment administration and side effects of hepatitis C treatments. The study was conducted on 182 participants and applying general population valued health states in time trade-off interviews with a 1-and 10-years time horizons ${ }^{(25)}$. A utility increment, assigned to patients who achieved SVR. This was derived from a multicenter, randomized, controlled, non-blinded trial, assessing the efficacy of the combination therapy of interferon and RBV versus no treatment for 204 patients with $\mathrm{HCV}$ chronic infection ${ }^{(26)}$.

- Cost Items: The direct medical care costs of HCV (drug regime, monitoring, side events and complications), from the Health Care system perspective, have been obtained from the National Liver Institute database. They have been supplemented with the authors' institutions' available information (Table 1). Capital costs were not involved. Cost data for the base-case represented the public pattern to reflect the average circumstances in Egypt. A top down-costing approach was used to determine the costs. Thus, drug regimens' calculated costs were based on the prescribed drug doses, including clinical trial therapy duration and unit drug costs. Monitoring costs varied by treatment regimen and cirrhosis status. Costs of adverse event were estimated based on each event's incidence and the allied costs associated with the treatment protocol. This was based on the Egyptian drug-treatment algorithms ${ }^{(27)}$. This study adopted the Hospital perspective to maximize the population's health gains while representing the most efficient allocation of the finite resources available to Egyptian government hospitals. Following the Egyptian

\section{RESULTS}

Table (1): Model input parameters guidelines, the applied discount rate (to all costs and health consequences) was $3.5 \%$ annually ${ }^{(28)}$.

- Time Horizon: The present cohort members transitioned between predetermined health states in yearly cycles and were followed for 37 years (based on Egypt's average life expectancy to capture the detailed events occurring during the course of disease ${ }^{(27)}$. With every cycle, the patients could remain in their current health state or could experience the following: fibrosis score $F 4$, DCC, HCC, L.T., or death from any cause ${ }^{(29)}$.

Phase III: Two incremental cost-effectiveness ratios (ICER) were calculated for screening versus noscreening scenarios among both cohorts; High-Risk population and Population with Low risk of acquiring $\mathrm{HCV}$ infection.

Phase IV: A two-day capacity-building workshop on Policy Brief Writing was held on 13th and 14th November at National Liver Institute in Cairo. The instructor was a reputable political science and public policy researcher at The American University in Cairo. Fifteen young to middle age researchers, actively participated, all of them are Master and/or M.D. holders of Public Health.

Data collection tools: CAPS quality checklists for critical appraisal of cohort and RCT and systematic literature reviews were used to ensure the quality of the used studies for retrieving data.

Data management and statistical analysis: The data were collected on Microsoft Excel sheets. Data were entered, cleaned, and revised through Microsoft Excel 2010 software. Then It was presented in tables and graphs. The primary data analysis was done using Microsoft Excel and SPSS. The decision-making tree and Markov Model were constructed using Microsoft Excel software for Window 10.

Quality Control and monitoring: Attendance sheets for the capacity building workshop were completed, including the participants' names, their I.D.s. The workshop successfully ended by preparing a policy paper for advocating for screening of the whole population. Deterministic and Probabilistic sensitivity analyses were done using Tree age software.

\section{Ethical Consideration:}

The study was approved by the Ethics Board of AlAzhar University and an informed written consent was taken from each participant in the study. This work has been carried out in accordance with The Code of Ethics of the World Medical Association (Declaration of Helsinki) for studies involving humans.

\begin{tabular}{|c|c|c|}
\hline Parameter & $\begin{array}{c}\text { Base } \\
\text { Case }\end{array}$ & $\begin{array}{c}\text { Range } \\
\text { Source of } \\
\text { data }\end{array}$ \\
\hline
\end{tabular}


https://ejhm.journals.ekb.eg/

\begin{tabular}{|c|c|c|c|c|}
\hline & & $\begin{array}{l}\text { Lower } \\
\text { Limit }\end{array}$ & $\begin{array}{l}\text { Upper } \\
\text { Limit }\end{array}$ & \\
\hline \multicolumn{5}{|l|}{$\begin{array}{l}\text { Population-related model Probabilities } \\
\end{array}$} \\
\hline 1- General populations: with relatively low risk of exposure to HCV & 0.119 & 0.111 & 0.126 & [18] \\
\hline 2- Populations at high risk: e.g. people who inject drugs & 0.556 & 0.494 & 0.617 & [18] \\
\hline Annual screening rate (no screening arm) & 0.05 & 0.045 & 0.055 & {$[20]$} \\
\hline Easy to treat (according to Egyptian classification) & 0.98 & 0.96 & 1 & [19] \\
\hline Sensitivity of (ELISA) used for HCV screening & $98.6 \%$ & 0.96 & 1 & [30] \\
\hline The proportion of Eligible patients to $\mathbf{t t t}$ (according to Egyptian classification) & 0.82 & 0.79 & $\mathbf{0 . 8 5}$ & [31] \\
\hline $\begin{array}{l}\text { The proportion of No SVR } 12 \text { among Easy to treat (non-response (according to } \\
\text { Egyptian classification) }\end{array}$ & 0.035 & & & {$[20]$} \\
\hline $\begin{array}{l}\text { The proportion of SVR among difficult to treat (according to Egyptian } \\
\text { classification) }\end{array}$ & 0.9 & & & [20] \\
\hline \multicolumn{5}{|l|}{$\begin{array}{l}\text { The proportion of different fibrosis states among chronic infected HCV } \\
\text { patients }\end{array}$} \\
\hline Fo & 0.199 & & & [31] \\
\hline F1 & 0.263 & & & \\
\hline F2 & 0.177 & & & \\
\hline F3 & 0.1 & & & \\
\hline F4 & 0.261 & & & \\
\hline \multicolumn{5}{|l|}{ Natural history of CHC- Transition probabilities } \\
\hline F0 to F1 & 0.117 & 0.041 & 0.155 & [32] \\
\hline F1 to F2 & 0.085 & 0.044 & 0.111 & [32] \\
\hline F2 to F3 & 0.12 & 0.092 & 0.201 & [32] \\
\hline F0, F1, F2 to Death & 0.001 & & & [32] \\
\hline F3 to F4 & 0.16 & 0.13 & 0.19 & [23] \\
\hline F3 to $\mathrm{HCC}$ & 0.001 & 0.0001 & 0.002 & {$[23]$} \\
\hline F3 to Death & 0.079 & 0.06 & 0.1 & [23] \\
\hline F4 to DCC & 0.039 & 0.03 & 0.05 & {$[22]$} \\
\hline F4 to $\mathrm{HCC}$ & 0.027 & 0.02 & 0.02 & [22] \\
\hline F4 to Death & 0.1 & 0.08 & 0.12 & [23] \\
\hline DCC to HCC & 0.02 & 0.018 & 0.21 & {$[22]$} \\
\hline DCC to LT & 0.05 & 0.04 & 0.06 & [22] \\
\hline DCC to Death & 0.26 & 0.21 & 0.31 & {$[22]$} \\
\hline HCC to LT & 0.15 & 0.12 & 0.18 & {$[22]$} \\
\hline HCC to Death & 0.43 & 0.38 & 0.48 & [23] \\
\hline L.T. ( 1 y) to death & 0.14 & 0.139 & 0.142 & [22] \\
\hline L.T. ( 2 y) to death & 0.057 & 0.05 & 0.06 & [22] \\
\hline \multicolumn{5}{|l|}{ Transitionprobabilitiesfor cirrhotic patients (without SVR) } \\
\hline $\mathrm{F} 4 \rightarrow \mathrm{DCC}$ & 0.031 & 0.0248 & 0.0372 & [23] \\
\hline $\mathrm{F} 4 \rightarrow \mathrm{HCC}$ & 0.027 & 0.0216 & 0.0324 & [23] \\
\hline \multicolumn{5}{|l|}{ Transitionprobabilitiesfor cirrhotic patients (with SVR) } \\
\hline $\mathrm{F} 4 \rightarrow \mathrm{DCC}$ & 0.001 & 0.0001 & 0.002 & [23] \\
\hline $\mathrm{F} 4 \rightarrow \mathrm{HCC}$ & 0.008 & 0.006 & 0.01 & [23] \\
\hline \multicolumn{5}{|l|}{ Utilities of Health States } \\
\hline F0, F1, F2 & 0.79 & 0.87 & 0.72 & [33] \\
\hline F3 & 0.85 & 0.66 & 0.96 & [22] \\
\hline F4 & 0.79 & 0.46 & 0.95 & [22] \\
\hline DCC & 0.72 & 0.26 & 0.91 & {$[22]$} \\
\hline $\mathrm{HCC}$ & 0.72 & 0.15 & 0.95 & {$[22]$} \\
\hline
\end{tabular}




\begin{tabular}{|l|c|c|c|c|}
\hline LT & $\mathbf{0 . 6 5}$ & $\mathbf{0 . 5 9}$ & $\mathbf{0 . 7 2}$ & {$[24]$} \\
\hline Post L.T. & $\mathbf{0 . 8 3}$ & $\mathbf{0 . 6 4}$ & $\mathbf{0 . 9 5}$ & {$[22]$} \\
\hline Utility decrement during treatment & $\mathbf{0 . 1 7}$ & $\mathbf{0 . 0 4}$ & $\mathbf{0 . 1 9}$ & {$[22]$} \\
\hline Utility increment for achieving SVR & $\mathbf{0 . 0 5}$ & $\mathbf{0 . 0 4 7}$ & $\mathbf{0 . 0 6}$ & {$[26]$} \\
\hline \multicolumn{1}{|c|}{ Annual costs of health states } & & & & \\
\hline Fibrosis scoreF3 & $\mathbf{4 , 0 0 0}$ & $\mathbf{3 2 0 0}$ & $\mathbf{4 8 0 0}$ & {$[\mathbf{2 7}]$} \\
\hline Fibrosis scoreF4 & $\mathbf{4 7 9 7}$ & $\mathbf{3 8 8 3 8}$ & $\mathbf{5 7 5 6}$ & {$[\mathbf{2 7}]$} \\
\hline DCC & $\mathbf{2 1 , 8 3 2}$ & $\mathbf{1 7 4 5 8}$ & $\mathbf{2 6 1 8 8}$ & {$[\mathbf{2 7}]$} \\
\hline HCC & $\mathbf{3 0 7 5 0}$ & $\mathbf{2 4 6 0 0}$ & $\mathbf{3 6 9 0 0}$ & {$[\mathbf{2 7}]$} \\
\hline LT & $\mathbf{2 5 0 0 0 0}$ & $\mathbf{2 0 0 0 0 0}$ & $\mathbf{3 0 0 0 0 0}$ & {$[\mathbf{2 7}]$} \\
\hline Post LT & 30,000 & 24,000 & 36,000 & {$[\mathbf{2 7}]$} \\
\hline Costs of monitoring & & & & \\
\hline Cost ofalfafetoprotein/1m & $\mathbf{4 0}$ & $\mathbf{3 2}$ & $\mathbf{4 8}$ & {$[\mathbf{2 7}]$} \\
\hline Cost ofPCR/3mo(0,3,6) & $\mathbf{3 5 0}$ & $\mathbf{2 8 0}$ & $\mathbf{4 2 0}$ & {$[\mathbf{2 7}]$} \\
\hline Cost of fibroscan/ultrasound/3mo & $\mathbf{2 0 0}$ & $\mathbf{1 6 0}$ & $\mathbf{2 4 0}$ & {$[\mathbf{2 7}]$} \\
\hline Cost ofINR/3mo & $\mathbf{3 0}$ & $\mathbf{2 4}$ & $\mathbf{3 6}$ & {$[\mathbf{2 7}]$} \\
\hline Cost ofserumalbumin/3mo & $\mathbf{1 2}$ & $\mathbf{1 0}$ & $\mathbf{1 4}$ & {$[\mathbf{2 7}]$} \\
\hline Cost ofbilirubin/3mo & $\mathbf{2 7}$ & $\mathbf{2 2}$ & $\mathbf{3 2}$ & {$[\mathbf{2 7}]$} \\
\hline Cost ofSGOT/3mo & $\mathbf{1 4}$ & $\mathbf{1 1}$ & $\mathbf{1 7}$ & {$[\mathbf{2 7}]$} \\
\hline Cost ofSGPT/3mo & $\mathbf{1 4}$ & $\mathbf{1 1}$ & $\mathbf{1 7}$ & {$[\mathbf{2 7}]$} \\
\hline Cost ofcreatinine/1mo & $\mathbf{2 0}$ & $\mathbf{1 6}$ & $\mathbf{2 4}$ & {$[\mathbf{2 7}]$} \\
\hline Cost ofCBC/3mo & $\mathbf{2 5}$ & $\mathbf{2 0}$ & $\mathbf{3 0}$ & {$[\mathbf{2 7}]$} \\
\hline Total costofmonitoringbeforetreatment/first year & $\mathbf{7 3 2}$ & $\mathbf{5 8 6}$ & $\mathbf{8 7 8}$ & {$[\mathbf{2 7}]$} \\
\hline Annual totalcostofmonitoring/secondyear2 & $\mathbf{2 1 9 6}$ & $\mathbf{1 7 5 7}$ & $\mathbf{2 6 3 5}$ & {$[\mathbf{2 7}]$} \\
\hline cost of screening & $\mathbf{9 0}$ & 80 & 100 & {$[\mathbf{2 7}]$} \\
\hline Costs of interventions & & & & \\
\hline SOF+LDV & $\mathbf{3 0 0 0}$ & $\mathbf{2 4 0 0}$ & $\mathbf{3 6 0 0}$ & {$[33]$} \\
\hline Discount Rate of Costs and QALYs & $\mathbf{0 . 0 3 5}$ & & & {$[28]$} \\
\hline
\end{tabular}

Table (1) presented the clinical parameters as follows: Mutually exclusive health states were studied: - Fo, defined as Normal liver of infected HCV patients, minimal fibrosis in the portal areas, and central veins' walls.

- F1, chronic hepatitis results in fibrous expansion of portal tracts, which may maintain a rounded contour or develop short spike-like septa involving only a few portal tracts.

- F2, chronic hepatitis eventually involves all portal tracts (34).

- Fibrosis score $\boldsymbol{F 3}$, identified as patients who had HCV infection without developing cirrhosis,

- Fibrosis score $\boldsymbol{F 4}$, identified as patients who had HCV infection with simultaneous cirrhosis.

- Patients in DCC are at high risk of mortality due to ascites, bleeding varices, encephalopathy, and jaundice. Whereas, in $\boldsymbol{H C C}$, which is defined as primary liver cancer that develops on top of chronic liver disease. - L.T., which was known by patients who had liver replacement due to life-threatening decompensated complications $^{(32)}$.

- Death which was defined as people died from any liverrelated causes ${ }^{(33)}$. Moreover, several assumptions were articulated providing comprehensiveness for the model. Initially, the population in the model were considered to be treatment-naive. Second, all infections were caused by HCV Genotype IV. Third, all chronic HCV patients, offered antiviral therapy would be treated with SOF and LDV. Fourth, we assumed HCV patients who achieved SVR would not develop relapse to No SVR ${ }^{(30,31)}$. All model input variables, their ranges, and sources are listed in Table (1). The sensitivity of the third-generation enzyme-linked immunosorbent assay (ELISA) used for HCV screening is $98.6 \%$, followed by confirmation with polymerase chain reaction (PCR) with $100 \%$ sensitivity (30). HCV infection prevalence rate among the general populations and high-risk population were retrieved from a meta-analysis and systematic review investigating the epidemiology of hepatitis $C$ virus in Egypt, $0.119( \pm 0.06)$ and $0.556( \pm 0.062)^{(18)}$. The Transition probabilities of $\mathrm{HCV}$ disease in patients with and without $S V R$, were extracted from a previous decision-analytic model that compared the cost-effectiveness of telaprevir added to pegIFN-2a and RBV (P.R.) compared to P.R. alone in adults with chronic HCV genotype $1^{(23)}$.

Regarding costs, drug regimens' calculated costs were based on the prescribed drug doses, including clinical trial therapy duration and unit drug costs. Monitoring costs varied by treatment regimen and cirrhosis status. Costs of adverse event were estimated based on each event's incidence and the allied costs associated with the treatment protocol .

The total cost of monitoring patients before treatment/first year, including the cost of PCR every 3 months, cost of fibroscan /ultrasound every 3 months, cost of serum 
albumin/3months, etc., was estimated to be 732 EGY ( \pm 146). The total discounted costs of the 2 alternative

Table (2): Costs, consequences and Incremental CostEffectiveness Ratio (ICER) of "Policy I" screening versus no-screening among populations at high risk of $\mathrm{HCV}$ infection

\begin{tabular}{|l|c|c|c|}
\hline Policy I & $\begin{array}{c}\text { Total } \\
\text { Discounted } \\
\text { costs (EGY) }\end{array}$ & $\begin{array}{l}\text { Total } \\
\text { Discounted } \\
\text { QALYs }\end{array}$ & ICER \\
\cline { 1 - 3 } Screening & 5687357.42 & 1367.06 & 3895.31 \\
\hline $\begin{array}{l}\text { No } \\
\text { screening }\end{array}$ & 513815.33 & 39.17 & \\
\hline
\end{tabular}

Concerning Policy (I) of High-Risk population, total discounted costs of the "Screen and Treat" scenario were estimated to be 5687357.42 EGY compared to 513815.33 EGY for the "No-screening" scenario. The total discounted QALYs for the "Screen and Treat" scenario was 1367.06 QALYs compared to 39.17 QALYs for the "No-screening" scenario. The calculated "Incremental Cost-Effectiveness Ratio" for this Policy was 3895.31 EGY/ QALY, where it is considered costeffective as it is below the Egyptian Threshold of CostEffectiveness Ratio determined to be 1 GDP/ capita, approx. $46000 \mathrm{EGY/} \mathrm{QALY.}$ policies and the corresponding outcomes as experienced QALY values were recorded.

Table (3): Costs, consequences and Incremental CostEffectiveness Ratio (ICER) of "Policy II" screening versus no-screening among general populations at low risk of $\mathrm{HCV}$ infection

\begin{tabular}{|c|c|c|c|}
\hline Policy II & $\begin{array}{l}\text { Total } \\
\text { Discounted } \\
\text { costs (EGY) }\end{array}$ & $\begin{array}{l}\text { Total } \\
\text { Discounted } \\
\text { QALYs }\end{array}$ & ICER \\
\hline Screening & 1513586.71 & 1125.42 & \multirow{2}{*}{$-\overline{364.64}$} \\
\hline $\begin{array}{l}\text { No } \\
\text { screening }\end{array}$ & 1921742.56 & 6.10 & \\
\hline
\end{tabular}

Concerning the Policy (II) of General population, total discounted costs of the "Screen and Treat" scenario were estimated to be 1513586.61 EGY compared to 1921742.56 EGY for the "No-screening" scenario. The total discounted QALYs for the "Screen and Treat" scenario was 1125.42 QALYs compared to 6.10 QALYs for the "No-screening" scenario. The calculated "Incremental Cost-Effectiveness Ratio" for this Policy was -34.64 EGY/ QALY, considered Dominant (cost-saving strategy).

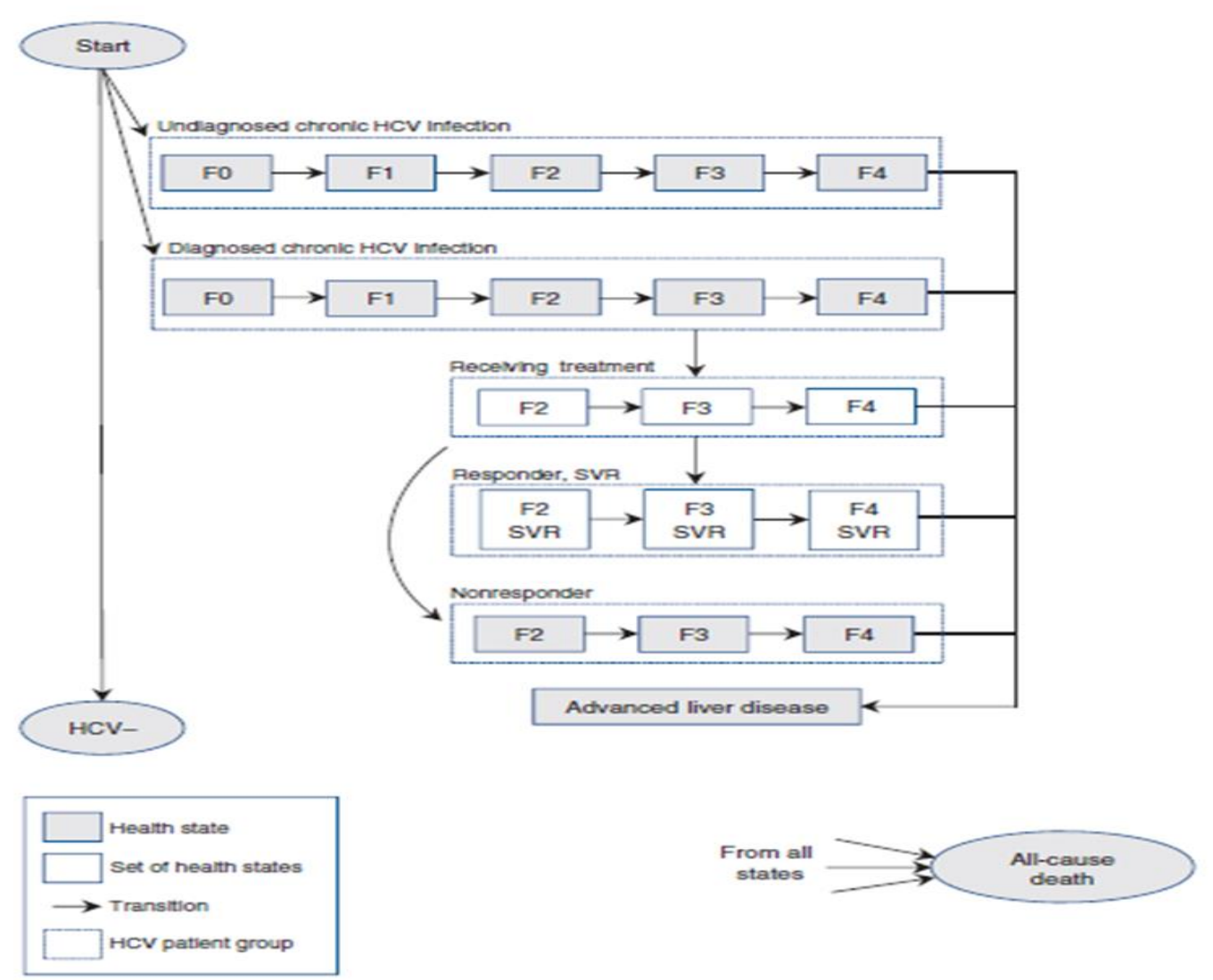


Fig (1): illustrating the used transition health states from F0 to F4 then progressing to advanced health state (DCC, HCC, and Liver transplant)

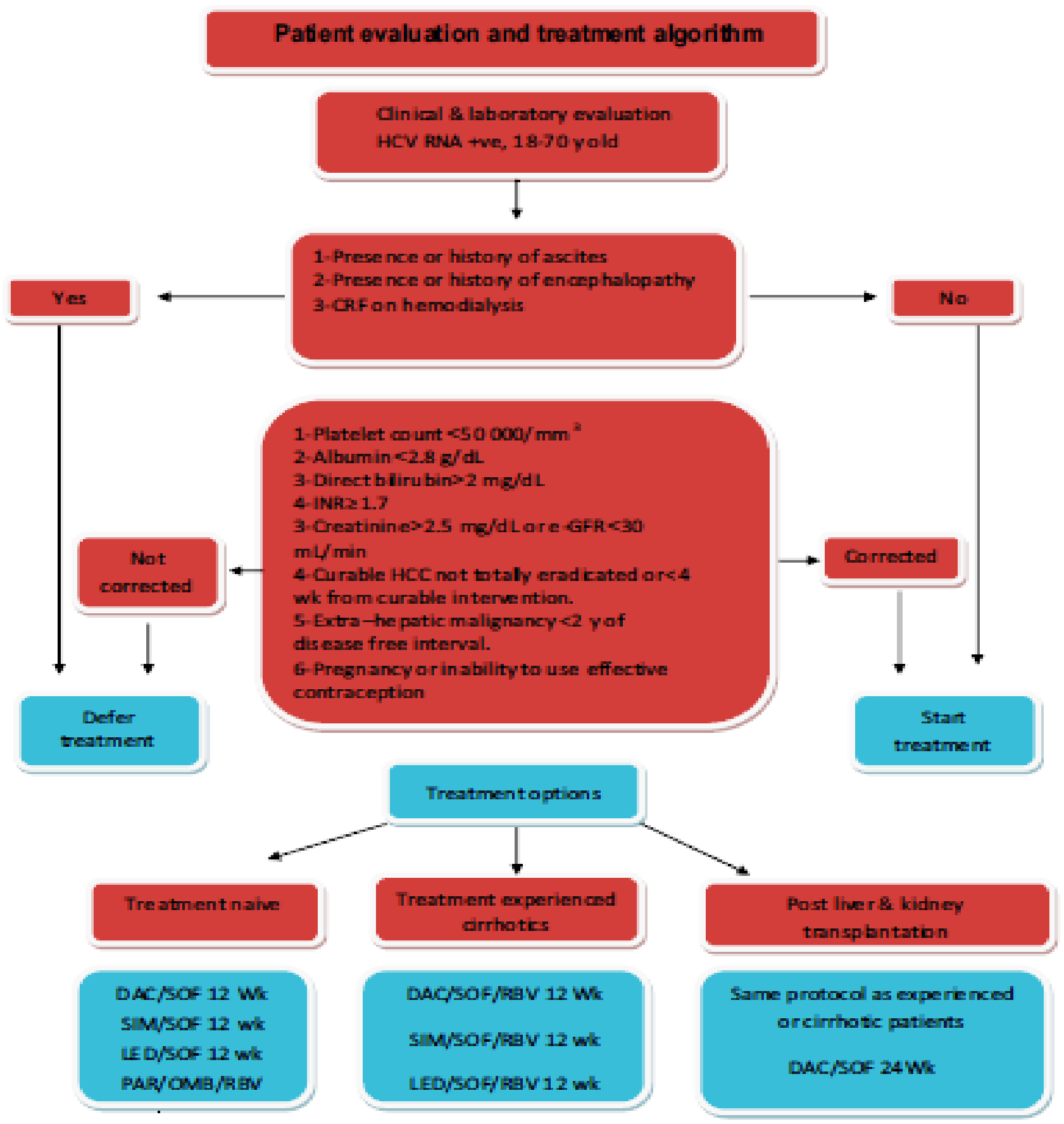

Fig (2): Journey of chronic hepatitis C virus (HCV) patient through the Egyptian HCV model of care. DAC: daclatasvir; SIM, simeprevir; LED, Ledipasvir; SOF, sofoprevir, REV, ribavirin, PAR, paritaprevir; OMB, ombitasvir

\section{DISCUSSION}

Egypt is one of the top ranked country according to the prevalence of hepatitis $\mathrm{C}$ in the emerging world. The persent study highlighted that applying hepatitis $\mathrm{C}$ screening and treatment for asymptomatic, average-risk Egyptian adults would save the general population and be highly cost-effective among high-risk populations.
Globally, high HCV prevelance countries with, reduced treatment costs, and achieving better SVR rate after treatment, found it cost-effective to implement $\mathrm{HCV}$ screening and treatment programs. Following the new drug registration in Egypt, the short-term plans are to focus on treating HCV patients having liver cirrhosis, who were identified in the past few years, followed by 
screening and treatment programs for the at-risk groups. Finaly, national screening and treatment of patients from the general population, would follow. On the national level, screening and treatment for $\mathrm{HCV}$ in Egypt could have substantial costs, with corresponding considerable health benefits. Comparing "No screening" versus "Screen-and-treat with DAA for the high-risk population in Egypt. The screening and treatment program would have cost 5687357.42 EGY compared to 513815.33 EGY for the "No-screening" scenario. saving 1367.06 QALYs in "Screen and Treat" scenario compared to 39.17 QALYs in "No-screening" scenario.

Regarding the general population's implementation, costs of "Screen and Treat" scenario were estimated to be 1513586.61 EGY compared to 1921742.56 EGY for the "No-screening" scenario. The total discounted QALYs of the "Screen and Treat" scenario was 1125.42 QALYs compared to 6.10 for the "No-screening" scenario. This finding was following the study done in Canada, where hepatitis $\mathrm{C}$ screening and management program saves lives and is costeffective, reaching from \$31 468/QALY to \$34 614/QALY gained over the lifetime of the cohort. Furthermore, the most cost-effective screening policies are: the strategy for the populations of immigrants who have high prevalence (scenario 2), the birth cohort with age group 25-64 years (scenario 3), and the birth cohort aged 45-64 years (scenario 4). On the other hand, screening and treatment programs targeting very lowrisk populations (e.g., the prevalence of $0.2 \%$ ) would be only marginally cost-effective, at $\$ 50$ 490/QALY gained over the lifetime of the cohort ${ }^{(33)}$. The main limitations of the present study was driving the clinical progression data of $\mathrm{HCV}$ infection to chronic liver diseases from international literature and reliance on clinical experts' opinions. Having more information on this for Egyptian patients would have added to the strength of Egypt's conclusions. Even though using Egyptian data is a strong point for making conclusions about Egypt, it also may be a limitation for making broader conclusions about other developing countries with intermediate-to-high prevalence. Additionally, due to the lack of data on future treatments for $\mathrm{HCV}$, we had to predict future events based on current data and treatment algorithms. Changes such as advances in treatment options or an unprecedented reduction of $\mathrm{HCV}$ prevalence could change our results.

\section{Conclusion:}

Our study is the first modeling study in Egypt to investigate the potential effects on the health system, examining 2 screening scenarios for diagnosing $\mathrm{HCV}$ infection on 2 population subgroups based on their risk of acquiring infection. The calculated "Incremental Cost-
Effectiveness Ratio" for implementing a high-risk population policy screening program was $3895.31 \mathrm{EGY/}$ QALY. It is considered cost-effective as it is below the Egyptian Threshold of Cost-Effectiveness Ratio determined to be $1 \mathrm{GDP} /$ capita, approx. $46000 \mathrm{EGY/}$ QALY. Furthermore, Policy II is concerned with implementing HCV screening on the general population. The calculated "Incremental Cost-Effectiveness Ratio" was -34.64 EGY/ QALY. It is considered dominant (costsaving strategy). Our results came following the National Screening Program of HCV launched in 2018 that aims at screening 62 million adults and 15 million adolescents by 2020.

\section{RECOMMENDATIONS}

\section{A-On Macro level (National level):}

1. According to this study, continuing the National HCV screening (on General population) is proven to be cost saving (Dominant strategy compared to no screening program), thus providing more QALYs and less cost.

2. More studies needed to be implemented in Egypt to measure the quality of life among Egyptians and studies that address the prevalence of complications.

\section{B-On Micro Level (Unit Level):}

It is recommended to conduct cost of illness studies on $\mathrm{HCV}$ infection, including all the probable health states they experience throughout infection. This could be through cost analysis of each preventive or curative service provided to $\mathrm{HCV}$ patients throughout their illness.

\section{REFERENCES}

1. Stanaway J (2016): The global burden of viral hepatitis from 1990 to 2013. Findings from the Global Burden of Disease Study 2013. Lancet, 6736 (16): 30579-7.

2. Ente Mohd Hanafiah K, Groeger J, Flaxman A et al. (2013): Global epidemiology of hepatitis $C$ virus infection: new estimates of age-specific antibody to HCV seroprevalence. Hepatology, 57: 1333-1342.

3. Lavanchy D (2011): Evolving epidemiology of hepatitis $\mathrm{C}$ virus. Clinical microbiology and infection: the official publication of the European Society of Clinical Microbiology and Infectious Diseases, 17: 107-115.

4. World Health Organization (2011): Hepatitis C. from http://www.who.int/mediacentre/factsheets/fs164/en/in dex.html. Accessed on April 24, 2017.

5. Perz J, Armstrong G, Farrington $L$ et al. (2006): The contributions of hepatitis $\mathrm{B}$ virus and hepatitis $\mathrm{C}$ virus infections to cirrhosis and primary liver cancer worldwide. J. Hepatol., 45 (4): 529-538.

6. Fung V, Brand R, Newhouse $\mathbf{J}$ et al. (2011): Using medicare data for comparative effectiveness research: opportunities and challenges. Am J. Manag Care, 17 (7): 488-96.

7. Ministry of Health and Population (2015): Egypt Health Issues Survey. (Cairo, Egypt and Rockville, 
Maryland, USA: Ministry of Health and Population and ICF International). https://dhsprogram.com/pubs/pdf/FR313/FR313.pdf

8. Jafferbhoy H, Gashau W, Dillon J (2010): Cost effectiveness and quality of life considerations in the treatment of hepatitis C infection. Clinicoecon Outcomes Res., 2: 87-96.

9. Ayoub H, Abu-Raddad L (2016): Impact of treatment on hepatitis $\mathrm{C}$ virus transmission and incidence in Egypt: A case for treatment as prevention. Journal of viral hepatitis, 31: 12-71.

10. Centers for Disease Control and Prevention (2017): Hepatitis .http://wwwnc.cdc.gov/travel/yellowbook/2014/chapter3-infectiousdiseases related-to-travel/hepatitis-c. Published July 10, 2015. Accessed on April 24, 2017.

11. World Health Organization (2016). Global health sector strategy on viral hepatitis 2016-2021. Towards ending viral hepatitis. https://apps.who.int/iris/handle/10665/246177

12. World Health Organization (2016). Combating hepatitis B and C to reach elimination by 2030: https://apps.who.int/iris/handle/10665/206453

13. Egyptian Ministry of Health and Population (2017): Plan of Action for the Prevention, Care \& Treatment of Viral Hepatitis, Egypt 2014-2018. http://www.emro.who.int/images/stories/egypt/VH_Pla n_of_Action_FINAL_PRINT1.pdf. Published 2014. Accessed on April 24, 2017.

14. Kim D (2015): Cost-effectiveness model for hepatitis $C$ screening and treatment: Implications for Egypt and other countries with high prevalence. Global public health, 10: 296-317.

15. McNeil J (2015): Curing Hepatitis $C$, in an Experiment the Size of Egypt. The New York Times, 16: 15-30.

16. Eckman M, Talal A, Gordon $S$ et al. (2013): Costeffectiveness of screening for chronic hepatitis C infection in the United States. Clin Infect Dis., 56 (10): 1382-1393.

17. Rein D, Smith B, Wittenborn J et al. (2012): The costeffectiveness of birth-cohort screening for hepatitis C antibody in U.S. primary care settings. Ann Intern Med., 156 (4): 263-270.

18. Kouyoumjian S, Chemaitelly $H$, Abu-Raddad $L$ (2018): Characterizing hepatitis $C$ virus epidemiology in Egypt: systematic reviews, meta-analyses, and metaregressions. Sci Rep., 8: 1661.

19. Wafaa Elakel, Manal H El-Sayed, Mohamed El Kassas et al. (2017): National treatment programme of hepatitis C in Egypt: Hepatitis C virus model of care. Journal of Viral Hepatitis, 24 (4): 10.
20. Asmaa Gomaa, Naglaa Allam , Imam Waked (2017). Hepatitis $C$ infection in Egypt: prevalence, impact and management strategies. Hepat Med., 9: 35.

21. Saab S, Gordon S, Park H et al. (2014): Costeffectiveness analysis of sofosbuvir plus peginterferon/ribavirin nin the treatment of chronic hepatitis $\mathrm{C}$ virus genotype1 infection. Aliment Pharmacol Ther., 40: 657-75.

22. Najafzadeh M, Andersson K, Shrank W et al. (2015): Cost-effectiveness of novel regimens for the treatment of hepatitis C virus. Ann Intern Med., 162: 407-19.

23. Brogan A, Talbird S, Thompson J et al. (2014): Costeffectiveness of telaprevir combination therapy for chronic hepatitis C. PLoS One, 9: e90295.

24. Hsu P, Federico C, Krajden M et al. (2012): Health utilities and psychometric quality of life inpatients with early and latest age hepatitis $\mathrm{C}$ virus infection. JGastroenterol Hepatol., 27: 149-57.

25. Matza L, Sapra S, Dillon J et al. (2015): Health state utilities associated with attributes of treatments for hepatitis C. Eur J Health Econ., 16: 1005-18.

26. Wright $M$, Grieve $\mathbf{R}$, Roberts $\mathbf{J}$ et al. (2006): Health benefits of antiviral therapy for mild chronic hepatitis $\mathrm{C}$ : randomized controlled trial and economic evaluation. HealthTechnolAssess, 10: 1-113.

27. Dalia Omran, Mohamed Alboraie and Mohamed EI Kassas (2018): Towards hepatitis $C$ virus elimination: Egyptian experience, achievements and limitations. World J Gastroenterol., 24 (38): 4330-4340.

28. Elsisi G, Kaló Z, Eldessouki $\mathbf{R}$ et al. (2013): Recommendations for reporting pharmacoeconomic evaluations in Egypt. Value Health Reg Issues, 2: 319-27.

29. Bedossa P, Poynard T (1996): The French METAVIR Cooperative Study Group. An algorithm for grading activity in chronic hepatitis C. Hepatology, 24: 289-293.

30. Veldt B, Saracco G, Boyer N et al. (2004): Long-term clinical outcome of chronic hepatitis $\mathrm{C}$ patients with sustained virological response to interferon monotherapy.Gut, 53: 1504-8.

31. Andrew J (2015): Cost-Effectiveness of Hepatitis C Treatment for Patients in Early Stages of Liver DiseaseHepatology, 61 (6): 1860-1869.

32. Thein H, Yi Q, Dore G, Krahn M (2008): Estimation of stage specific fibrosis progression rates in chronic hepatitis $\mathrm{C}$ virus infection: A meta-analysis and metaregression. Hepatology, 48: 418-31.

33. William W, Aysegul E, Jordan J et al. (2017): Modelbased projection of health and economic effects of screening for hepatitis C in Canada. CMAG., 5 (3): E662E672.

34. Goodman $Z$ (2007): Hepatiitis $C$ facts. Journal of Hepatology, 47: 598-607. 